\title{
Age-related changes of bone strength phenotypes: observational follow-up study of hand bone mineral density
}

\author{
I. Malkin • G. Bigman • R. Matias • L. Kalichman • \\ M. J. Seibel • E. Kobyliansky • G. Livshits
}

Received: 29 May 2006 / Accepted: 11 September 2006 / Published online: 23 November 2006

(C) International Osteoporosis Foundation and National Osteoporosis Foundation 2006

\begin{abstract}
Summary In a cross-sectional and follow-up study, we evaluated age-related changes of hand bone mineral density in both sexes using data obtained by digital radiographic densitometry in a large Chuvashian cohort.

Objectives The aim of the study was to evaluate age-related changes of hand bone mineral density (BMD) in both sexes using data obtained by digital radiographic densitometry in a large Chuvashian cohort.

Methods The data were gathered in 1994 (557 individuals) and 2002 (513 individuals). The latter sample included 260 individuals who were studied only during the second expedition and 253 individuals who had been previously investigated in 1994. Digital radiographic densitometry was employed to evaluate hand BMD. Statistical analyses included a maximum likelihood-based model-fitting technique.

Results and conclusions Cross-sectional study: Since the third decade of life, men lost hand BMD at all ages, but it remained higher than in women at any age. The most parsimonious and best-fitting piecewise linear models of
\end{abstract}

I. Malkin · G. Bigman • R. Matias • E. Kobyliansky •

G. Livshits $(\square)$

Human Population Biology Research Unit,

Department of Anatomy and Anthropology,

Sackler Faculty of Medicine,

Tel Aviv University,

Tel Aviv 69978, Israel

e-mail: idak@post.tau.ac.il

L. Kalichman

Department of Physical Therapy,

The Stanley Steyer School of Health Professions,

Tel Aviv University,

Tel Aviv, Israel

M. J. Seibel

Bone Research Program, ANZAC Research Institute,

Sydney, NSW, Australia age-related changes of hand BMD had higher prediction values in females than in males $\left(R^{2}=0.48-0.58\right.$ vs $R^{2}=$ $0.20-0.29$, correspondingly). The compact BMD is more sensitive to age changes than the total BMD in both sexes. Longitudinal study: Hand BMD loss was higher in males than in females aged 30-59, but afterwards this trend reversed. The highest loss in both sexes was in ages 50-59.

Keywords Bone mineral density - Digital radiographic densitometry $\cdot$ Repeated measurements

\section{Background}

Each year, about two million people worldwide suffer a bone fracture related to osteoporosis. In 1990, there were 1.6 million hip fractures per year worldwide and this number is estimated to reach 6 million by 2050 [1]. The pathogenesis of bone fragility is heterogeneous and many studies have suggested at least three bone characteristics that may be associated with osteoporotic fractures, namely bone mineral density (BMD), bone size and geometry [2], and bone turnover [3]. BMD may serve as an objective estimate of bone strength, predicting $60-70 \%$ of its variation [4]. As a result, BMD is a powerful and the most frequently used measurable determinant of osteoporotic fracture risk.

Currently, noninvasive bone densitometry is widely used to assess skeletal mineral status and estimate future fracture risk [5]. In the present study, we used the digital radiographic densitometry method to evaluate the average BMD of whole bone (BMD-total) as well as the compact part of bone (COMP). Evaluation of hand BMD is of particular interest since the hand phalanges undergo early age-related changes and they are very sensitive to bone 
resorption [6]. This method is similar to radiographic absorptiometry $[7,8]$, but it is rapid, easy to perform, and requires low radiation exposure. Bone densitometry can also be less expensive than dual X-ray absorptiometry (DXA) because it is very widely applicable and the initial investment is small. Thus, it may be used for population screening or preselection for further work-up in areas with little access to DXA scanners. The validity of the method was evaluated by Bouxsein et al. [8], who found that bone mineral content estimates by this method were strongly correlated $(r=0.89, p<0.001)$ with ash weight (the "gold standard" of bone mineral content evaluation).

Several studies evaluated the possibility of using digital radiographic densitometry for assessment of future fracture risk. Reed et al. [9] found that the correlation between the results of digital radiographic densitometry is comparable with that reported between DXA measurements at the forearm, spine, and hip. They also suggested that digital radiographic densitometry may provide a feasible method for the assessment of future fracture risk. Hagiwara et al. [10] also concluded that digital radiographic densitometry of the second metacarpal bone shows a gradient of risk for spinal fracture only slightly below that of forearm DXA.

Most investigations of age-related changes of BMD have been cross-sectional [11, 12], including ours [13, 14], and have not analyzed the longitudinal progression of bone loss. Several longitudinal, population-based studies described BMD change in women during the menopausal transition [15-18] and in old age [19-24]. However, few longitudinal, population-based surveys have evaluated these changes in men [25-27]. Studies comprising both sexes from the same population are even rarer, and those existing are from elderly populations [28-30], except a recently published one [31]. Moreover, some age-related parameters of BMD have not yet been determined with certainty, for example, the age of peak bone values and the age at which premenopausal bone loss commences [14, 32, 33]. Longitudinal, population-based studies describing BMD changes in both sexes from a young to an old age are therefore still lacking.

The aim of the present study was to evaluate age-related changes of hand skeleton BMD in both sexes using the data obtained by digital radiographic densitometry in a large Chuvashian cohort using cross-sectional and longitudinal study designs.

\section{Materials and methods}

Sample The subjects of the present study were Chuvashians, who live in numerous villages along the Volga River in the Chuvash Autonomy, Russian Federation. The data were gathered during two expeditions undertaken during August/September 1994 (557 individuals) and September
2002 (513 individuals) by the Department of Anatomy and Anthropology, Sackler Faculty of Medicine, Tel-Aviv University (Israel) and by the Anuchin Research Institute and Museum of Anthropology, Moscow State University (Russia). On the first expedition we collected data on 557 individuals, 303 males with a mean age of $46.2(18-84)$ and 254 females with a mean age of 50.7 (19-79). On the second expedition, data were collected on 513 individuals, 251 males with a mean age of $51.5(18-86)$ and 262 females with a mean age of 54.5 (18-84). Of these, 260 individuals were studied for the first time and 253 individuals had been previously investigated in 1994. The expeditions were part of a Chuvash Skeletal Aging Study (ChuSAS) project investigating different aspects of skeletal aging within the Chuvash population. The same team of investigators collected all the information and performed all measurements during both expeditions.

In the present study, we collected data from residents of a number of small villages located in the Volga region. A rural population is more homogeneous than urban in terms of ethnicity, occupation, and physical activity. Most participants shared similar socioeconomic conditions, agriculture being their principal source of livelihood. Families that live in this area have lived under the same environmental conditions for generations and were not exposed to an outside genetic flow [34]. Data from $80-90 \%$ of the families (including all family members who were living in the area at the time of the expedition) were obtained. Since almost every individual was related to one of the families, we were able to collect data on up to $90 \%$ of the population in each village. All studied individuals were recruited randomly, i.e., regardless of the readings of any of the measured variables.

The present research involved taking X-ray radiograms of hand bones, collecting the blood samples, anthropometry (weight, stature, etc.), and interviewing subjects. The collected information included the sex, age, occupation, and life style factors. Data on chronic morbidity and medical treatment were obtained from their medical records and were completed during the interview. The individuals with known bone disease or with amenorrhea were not included in the study. There were no women using hormone replacement therapy or taking glucocorticoid medicine in the sample. All participants signed informed consent forms before entering the study, which was approved by the Helsinki Ethics Committee of Tel-Aviv University.

Bone measurements and traits construction Plain radiographs of both hands were taken from each study participant using a standard radiographic technique, as described in detail by Livshits et al. [35]. The hands were placed on the same film-containing plate to avoid any film or development variation. Radiography of the hand was 
carried out in the presence of a compact bone wedge phantom for relating X-ray attenuation to bone mass that was placed between the two hands. The wedge had the length of a working surface of $100 \mathrm{~mm}$, width $10 \mathrm{~mm}$, and an angle between the upper and lower surface of $9^{\circ}$. The mineralization (area's BMD) of the wedge increased by $1 \mathrm{mg} / \mathrm{mm}^{2}$ per $4 \mathrm{~mm}$ of the wedge length, correspondingly, and the measurement range varied between 0 and $25 \mathrm{mg} /$ $\mathrm{mm}^{2}$. Using this type of phantom is critical for accurate radiographic evaluation of BMD [36].

All roentgenograms were digitized using a commercial flatbed scanner (UMAX 2100) with a transmission unit. The obtained digital gray-scale images were $1,200 \times 1,800$ pixels with a resolution of 150 DPI. The bone edge of each measured bone and the borders of the reference wedge were marked manually by a computerized contour line. Further analysis of the image data was automated. The local BMD was evaluated for each image pixel and then averaged for two different regions: BMD-total for the whole surface area of bone projection and COMP for the middle shaft area ( $40 \%$ of the bone length in the bone center) (Fig. 1).

The measurements were taken from the metacarpal bones, as well as the proximal and middle phalanges of the second, third, and fourth fingers of both hands (18 bones in total).

We evaluated the method's accuracy and reliability in two ways: (1) we randomly selected 30 X-rays and

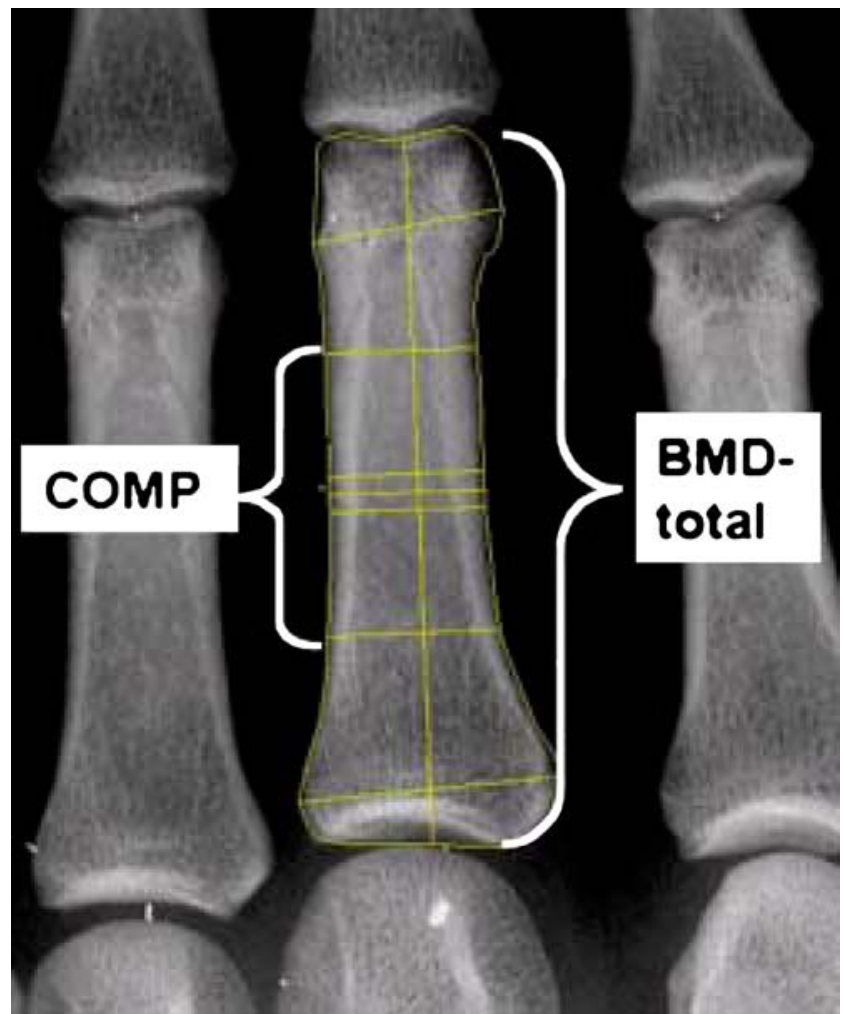

Fig. 1 Scheme of BMD evaluation: BMD-total was evaluated on whole surface area of bone projection and COMP on the middle shaft area $(40 \%$ of the bone length in the bone center) measured them twice 2 weeks apart, and (2) we assessed short-term precision, by measuring two X-rays ten times each, with repositioning between measurements. In all measurements, the investigator was blind to the results of previous measurements and to any identification indices. Relative error (RE) was estimated by dividing the mean square difference between sequential measurements by the mean value of the measurements. In the first approach of RE evaluation, BMD-total measurements varied between 3.1 and $3.8 \%$ in different sites; and in the second approach, the $\mathrm{RE}$ range was $1.3-2.4 \%$.

Statistical analysis Eighteen BMD-total and COMP measurements were highly significantly correlated with each other $(r=$ between 0.44 and $0.91, p<0.001)$, within each of the two groups of traits. Using STATISTICA version 5.5 software [37], we undertook principal component analysis of each of these trait groups to avoid the problem of multiple comparisons, redundancy of information, and repetition of measurement error. We used the original data regardless of the sex and age of the individual to capture as many common variations as possible, with an eigenvalue 1 criterion to retain the components. Since only one principal component was extracted in each analysis, there was no need for the further rotation procedure. The first principal components derived from the outcome of these analyses were used in further analyses.

The investigation of age-related changes of bone strength phenotypes was performed in a number of stages. First, we divided the collected data into four groups:

1. Measurements conducted in 1994 for individuals not repeatedly evaluated in 2002

2. Measurements performed in 1994 for individuals repeatedly evaluated in 2002

3. Measurements conducted in 2002 for individuals previously evaluated in 1994

4. Measurements evaluated in 2002 for individuals not evaluated in 1994

Box plots of those four groups for each of the studied variables were constructed. Independent groups 1, 2, and 4 were compared using one-way analysis of covariance (ANCOVA). The groups (2 and 3 ) with repeatedly measured individuals were compared using the paired $t$ test. At the second stage, we examined the aging pattern of BMD-total and COMP in the sample, combining groups 1, 2 , and 4 using the maximum likelihood estimator, as implemented in the statistical package MAN-6 [38]. This software enables the investigator to examine the fit of the various mathematical functions (models) for empirical data. Estimation of the model's parameters is based on the least mean squares method, subservient to the maximum likelihood method, assuming a normal distribution of the trait 
[14]. Specifically, we examined continuous piecewise polynomial functions of age (e.g., piecewise linear two, three, or four interval age dependence) as models for the population age-dependent mean. We adjusted our BMD data for age using the best-fitting and most parsimonious aging model. Then we investigated the dependence of ageadjusted BMD on body height and weight, smoking, alcohol consumption, and menopausal status (of women). In the next stage, the difference (delta scores) between two measurements taken 8 years apart was calculated for each individual measured twice (groups 2 and 3). The whiskers plot was used to assess the average rate of change in hand BMD in the different age groups (according to the decades of age). Using the model parameters obtained in the previous stage, we estimated the predicted 8-year change of BMD in individuals from the second group, separately for males and females. The predicted BMD values were compared to the observed BMD in the third group, using the $t$-test for repeated measurements.

Finally, we evaluated the possible population trend of peak bone mass. The group of individuals aged 35-42 (a proximal age of peak bone mass point) was tested for differences attributed to the sampling year (1994 or 2002) for each sex separately.

\section{Results}

The correlations between the measurements of the right and left hands were all statistically significant $(p<0.01)$ and varied between 0.74 and 0.89 for BMD-total and between 0.75 and 0.90 for COMP. In Table 1 we present the mean values and standard deviations of BMD-total of the studied bones of both hands in males $(\mathrm{M})$ and females $(\mathrm{F})$. The lowest mean BMD-total value was in the second proximal phalanx, both in females $\left(2.97 \pm 0.61 \mathrm{mg} / \mathrm{mm}^{2}\right)$ and in males $\left(3.61 \pm 0.55 \mathrm{mg} / \mathrm{mm}^{2}\right)$, and the highest was in the third middle phalanx (in females $4.81 \pm 0.78 \mathrm{mg} / \mathrm{mm}^{2}$ and in males $5.71 \pm 0.80 \mathrm{mg} / \mathrm{mm}^{2}$ ).

The main results of the principal component analyses are shown in Table 2, where the load scores for each trait within
Table 2 Principal component analyses of both study variables: load scores

\begin{tabular}{lcc}
\hline Hand bones $^{\mathrm{a}}$ & BMD-total & COMP \\
\hline Metacarpal bone, left 2 & 0.754 & 0.795 \\
Metacarpal bone, left 3 & 0.788 & 0.799 \\
Metacarpal bone, left 4 & 0.806 & 0.822 \\
Metacarpal bone, right 2 & 0.713 & 0.774 \\
Metacarpal bone, right 3 & 0.763 & 0.776 \\
Metacarpal bone, right 4 & 0.792 & 0.802 \\
Middle phalanx, left 2 & 0.873 & 0.868 \\
Middle phalanx, left 3 & 0.835 & 0.834 \\
Middle phalanx, left 4 & 0.838 & 0.834 \\
Middle phalanx, right 2 & 0.879 & 0.877 \\
Middle phalanx, right 3 & 0.845 & 0.831 \\
Middle phalanx, right 4 & 0.852 & 0.853 \\
Proximal phalanx, left 2 & 0.920 & 0.929 \\
Proximal phalanx, left 3 & 0.899 & 0.901 \\
Proximal phalanx, left 4 & 0.908 & 0.909 \\
Proximal phalanx, right 2 & 0.910 & 0.927 \\
Proximal phalanx, right 3 & 0.886 & 0.896 \\
Proximal phalanx, right 4 & 0.890 & 0.897 \\
Explained variance & 12.82 & 13.09 \\
Percent of the total variance & $71 \%$ & $72 \%$ \\
\hline
\end{tabular}

${ }^{\mathrm{a}} 2,3$, and 4 - second, third, and fourth fingers

each category are presented. The scores were generally high and in both analyses only one principal component with an eigenvalue $>1.0$ was retained. We therefore used the first principal component in further analyses.

As shown in the box plots (Fig. 2), there were virtually no differences between the mean values of both BMD variables in our three cross-sectional samples. However, there were obvious and statistically significant differences in the mean values of the BMD-total and COMP between these groups, on the one hand, and the third group, on the other hand (Fig. 2).

Using the likelihood ratio test, we chose the best-fitting and most parsimonious models among the entire set of empirical poly-interval regression models (Fig. 3). In males, for BMD-total the two-interval linear model was chosen. BMD-total increased ( 1.6 $\pm 0.6 \mathrm{SD}$ per 10 years) up to age $23.5( \pm 1.7)$ years, after which a trend of bone loss ( $-0.21 \pm 0.02$ SD per 10 years) was observed. For COMP

Table 1 Mean values (standard deviations) and [coefficients of variation] of BMD-total of studied hand bones of both hands in males (M) and females (F) separately ${ }^{\mathrm{a}}$

\begin{tabular}{|c|c|c|c|c|c|c|}
\hline \multirow{2}{*}{$\begin{array}{l}\text { Ray } \\
\text { Bone }\end{array}$} & \multicolumn{2}{|l|}{ 2nd } & \multicolumn{2}{|l|}{$3 \mathrm{rd}$} & \multicolumn{2}{|l|}{4 th } \\
\hline & M & $\mathrm{F}$ & M & $\mathrm{F}$ & M & $\mathrm{F}$ \\
\hline Metacarpal & $4.44(0.87)[0.20]$ & $3.80(0.81)[0.21]$ & $4.49(0.75)[0.17]$ & $3.90(0.77)[0.20]$ & $4.14(0.80)[0.19]$ & $3.57(0.80)[0.22]$ \\
\hline Proximal phalanx & $3.65(0.55)[0.15]$ & $2.97(0.61)[0.21]$ & $4.33(0.72)[0.17]$ & $3.66(0.86)[0.23]$ & $4.10(0.67)[0.16]$ & $3.37(0.66)[0.20]$ \\
\hline Middle phalanx & $4.90(0.67)[0.14]$ & $4.09(0.76)[0.19]$ & $5.71(0.80)[0.14]$ & $4.81(0.78)[0.16]$ & $5.48(0.81)[0.15]$ & $4.45(0.78)[0.18]$ \\
\hline
\end{tabular}

${ }^{\text {a }}$ Values in $\mathrm{mg} / \mathrm{mm}^{2}$ 
Fig. 2 Box plot (mean \pm standard error and $95 \%$ confidential interval) of BMD-total and COMP of four studied groups. Group 1: measurements evaluated in 1994 for individuals not evaluated in 2002; group 2: measurements evaluated in 1994 for individuals evaluated repeatedly in 2002; group 3: measurements evaluated in 2002 for individuals previously evaluated in 1994; and group 4: measurements evaluated in 2002 for individuals not evaluated in 1994

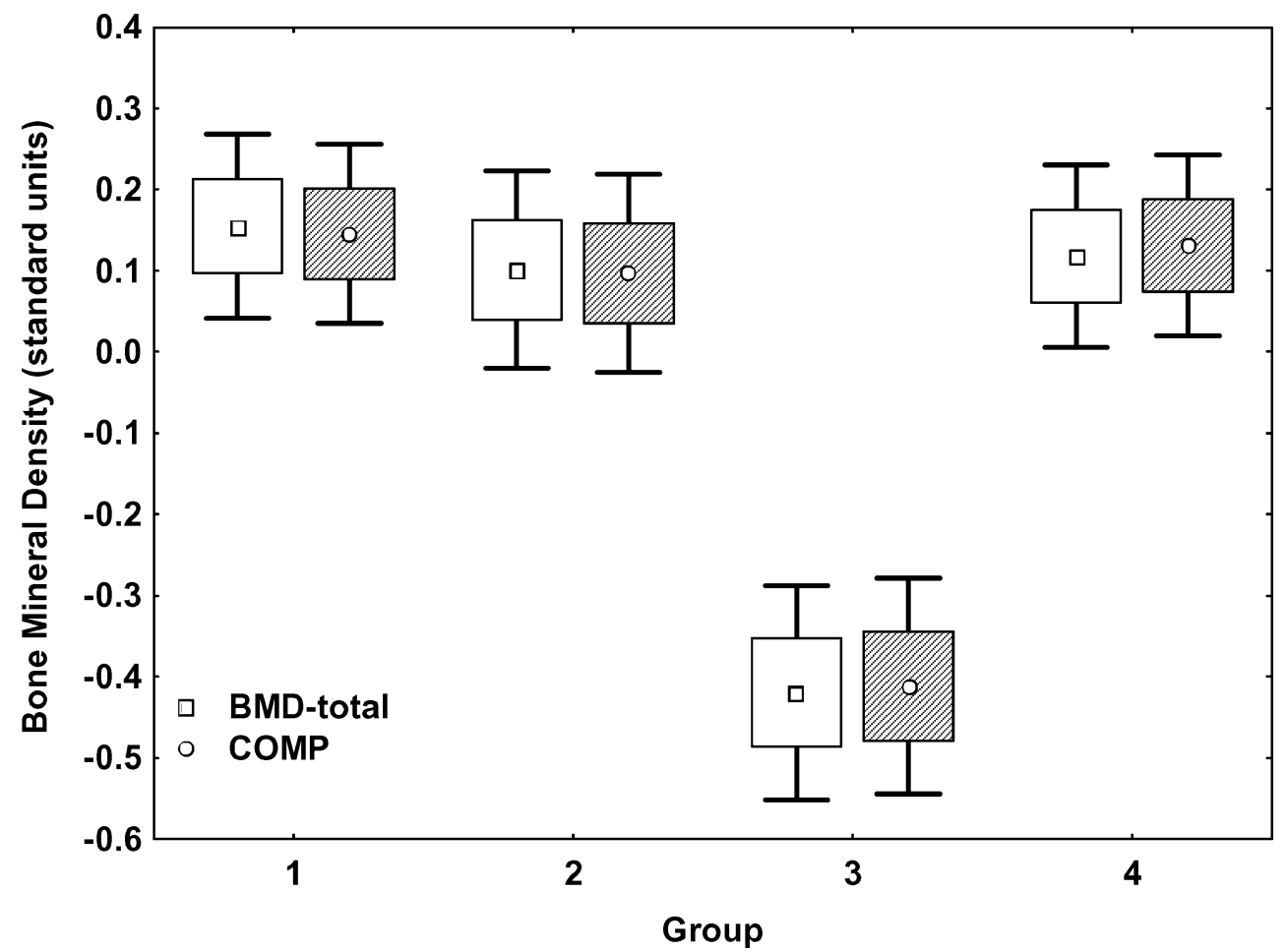

the most parsimonious model was the three-interval model: an increase $(\sim 0.74 \pm 0.31$ SD per 10 years $)$ up to age 26.4 $( \pm 2.4)$, a slow trend of bone loss $(\sim-0.15 \pm 0.06$ SD per 10 years) up to age $49.0( \pm 2.0)$, and after that a stronger trend of BMD decrease ( $-0.36 \pm 0.06 \mathrm{SD}$ per 10 years). The difference in the rate of changes between the two last intervals was significant, according to the likelihood ratio test at $p=$ 0.05 . The age dependence explained about $20 \%$ of the total variance for BMD-total, whereas for COMP it was $29 \%$.

In females, for BMD-total the three-interval model was chosen, with a rapid increase $(\sim 0.35 \pm 0.07$ SD per 10 years $)$ of BMD-total up to the age of $44.4( \pm 1.5)$, after which the mean bone density begins to decrease rapidly $(\sim-0.88 \pm$ 0.08 SD per 10 years) until age $60.0( \pm 1.4)$. Afterwards, it continued to decrease but at a much lower rate $(\sim-0.35 \pm$ $0.09 \mathrm{SD}$ per 10 years). The difference in the last two rates was highly significant $(p<0.001)$. For COMP the fourinterval model was better than the three-interval model according to the likelihood ratio test at $p<0.05$. Again, we observed a rapid trait increase $(\sim 0.63 \pm 0.15$ SD per 10 years) up to age $30.8( \pm 1.9)$. After that there were no changes up to age $48.0( \pm 0.6)$, when the rapid decrease ( $-1.02 \pm 0.07 \mathrm{SD}$ per 10 years) begins and continues up to age $60( \pm 1.4)$. Afterwards the rate of decrease becomes lower $(\sim 0.38 \pm 0.08 \mathrm{SD}$ per 10 years $)$. In females, the age dependence explains 48 and $58 \%$ of the BMD-total and COMP variance, respectively.

Results of analysis of variance (ANOVA) indicated that there were no significant differences in age-adjusted BMD associated with smoking and alcohol consumption. We found no significant correlations between age-adjusted BMD and years since menopause in postmenopausal women $(N=159$, $p=0.83)$. In the multiple regression model we found that for males both height $(\beta=0.16, p=0.007)$ and weight $(\beta=0.12$, $p=0.04$ ) were significant and explained $6.5 \%$ of age-adjusted BMD variance. In females only height $(\beta=0.16, p=0.005)$ was found significant and explained $4.6 \%$ of the variance.

Figure 4 presents the mean values of hand BMD change in individuals measured in 1994 and 2002 (deltas) for each decade of age (in 1994) and according to sex. At ages 18 29 the mean amount of loss was nearly similar in males and females (0.18 and 0.23 SD, correspondingly). During the following two decades of life, the rate of BMD loss became consistently higher in both sexes, in particular in males. The highest rate of change was observed in the age group 50 59 , and in both sexes. Next, the rate of bone loss clearly slows down in both males and females. Interestingly, the relative extent of changes (delta) was usually higher in men than in women.

Results of the paired $t$-test that compared the predicted and observed BMD-total in the third group are shown in Fig. 5. There was no statistically significant difference between the two estimates in females $(t=1.08, d f=121, p=$ 0.28 ). However, in males the observed BMD values were significantly lower than estimates predicted from the aging curve $(0.49 \pm 0.08 \mathrm{SD}$ vs $0.72 \pm 0.08 \mathrm{SD} ; t=2.81, d f=106$, $p<0.01)$. To explain this discrepancy, we hypothesized the existence of the population trend regarding the peak bone 
Fig. 3 Scatter plots of age dependence for BMD-total and COMP for males (upper graphs) and females (lower graphs). The line shows the best-fitting model (piecewise linear) of the relationship between BMD and age
MEN

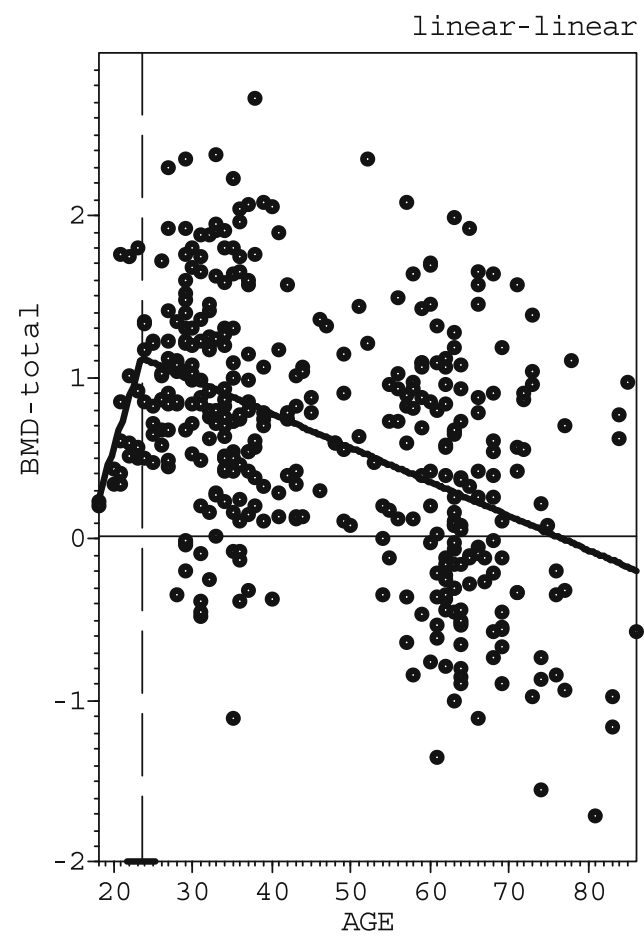

WOMEN

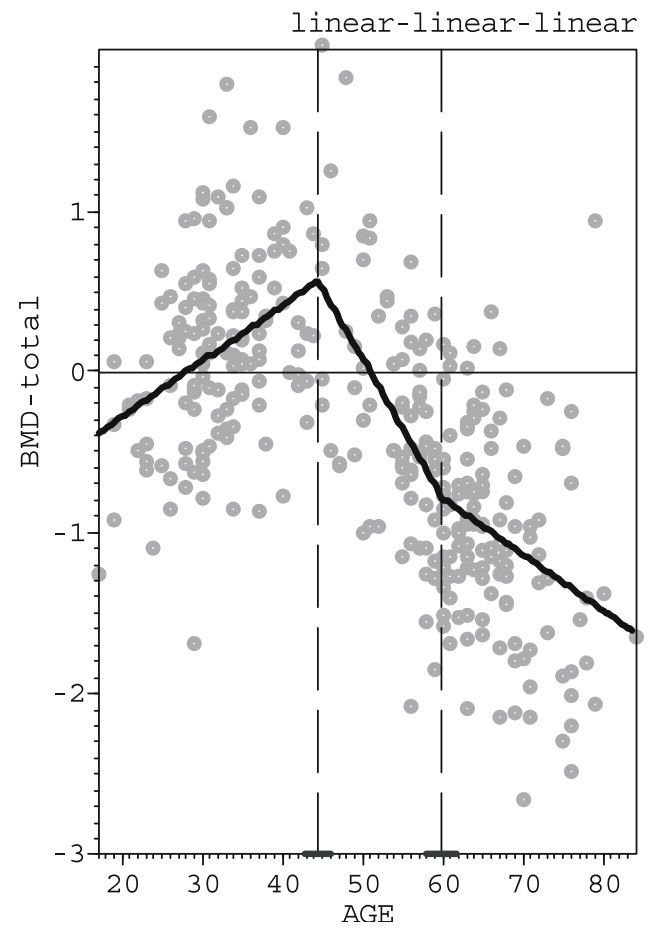

MEN

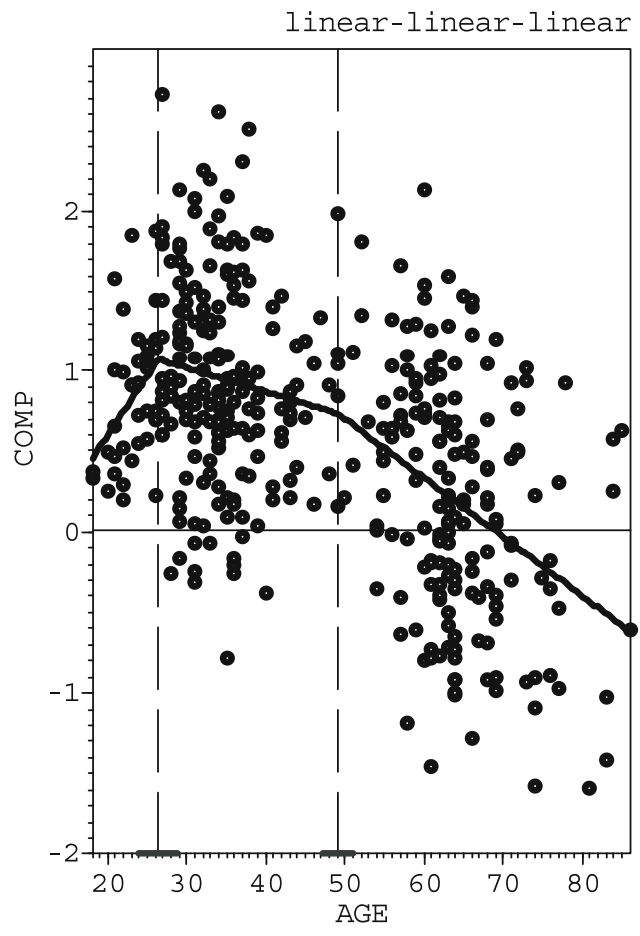

WOMEN

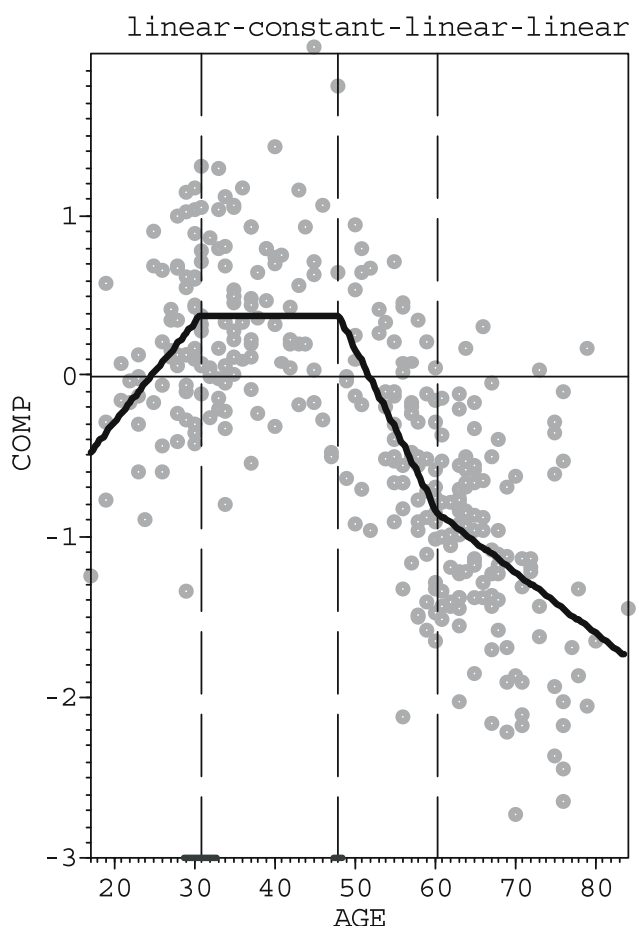

mass value. More specifically, we analyzed the group of individuals aged 35-42. The mean COMP value significantly differs ( $p=0.003$ for males and $p=0.001$ for females) between individuals sampled in 1994 and sampled in 2002. The mean value of COMP was significantly lower in groups of individuals sampled in 2002.

\section{Discussion}

The primary goal of this study was to examine the agerelated changes in BMD phenotypes in the peripheral skeleton, as assessed using digital radiographic densitometry. The age composition of the study sample allowed us to 
Fig. 4 Whisker plot of mean change (delta scores) \pm standard error (SE) of hand BMD over 8 years in males and females. Axis $x$ age groups, axis $y$ the mean values \pm standard error of BMD change

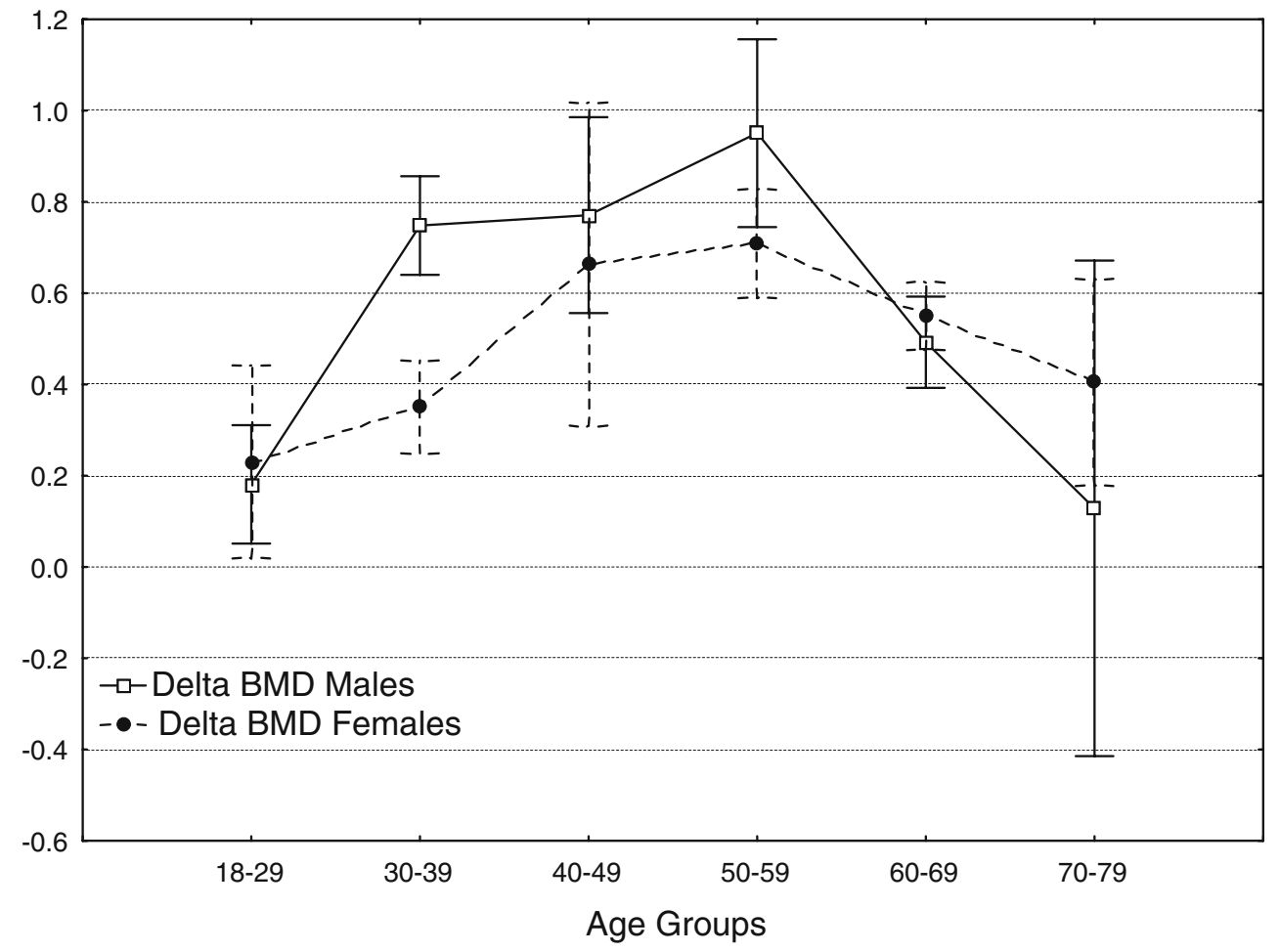

conduct this analysis over a wide range of ages. When groups 1,2, and 4, which were recruited randomly but at different times, were compared, we found no difference between their group means and distributions (Fig. 2), suggesting that the samples collected in the two expeditions, 8 years apart, were comparable and were taken from the same general population. The third group, which included individuals who were repeatedly measured, was statistically significantly different from the others, with mean values of BMD-total and COMP clearly lower than in the other groups.

It is worth mentioning that when the observed BMD data in the third group were compared to the estimates expected from the cross-sectional prediction, using the corresponding
Fig. 5 Results of paired $t$-test for comparison of the predicted BMD and observed BMD-total of the third group. Box-andwhisker plots (mean \pm standard error and standard deviation) of studied values for males and females

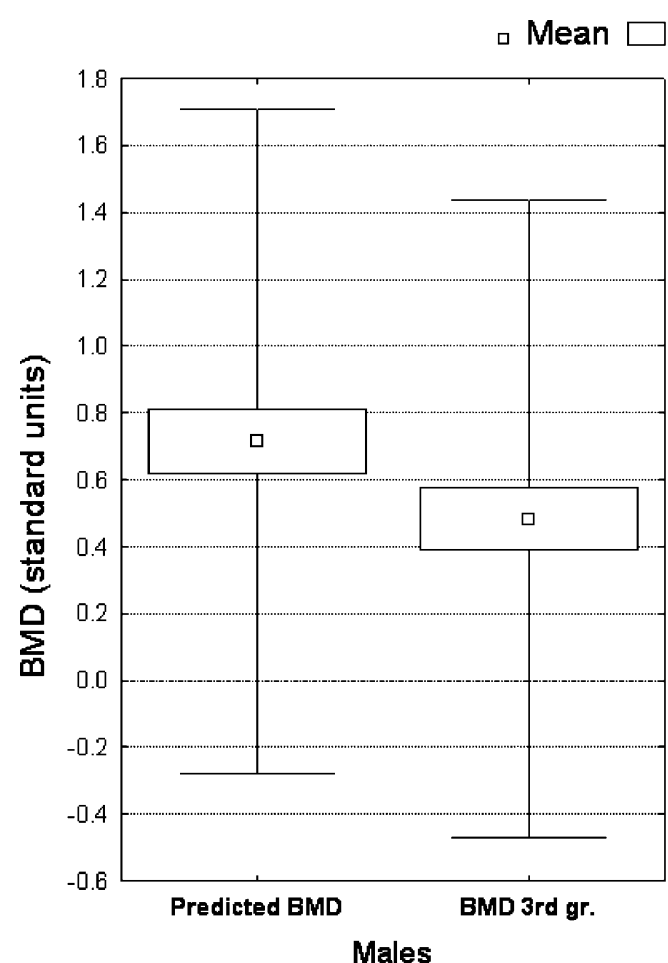

\pm SE 工 \pm SD

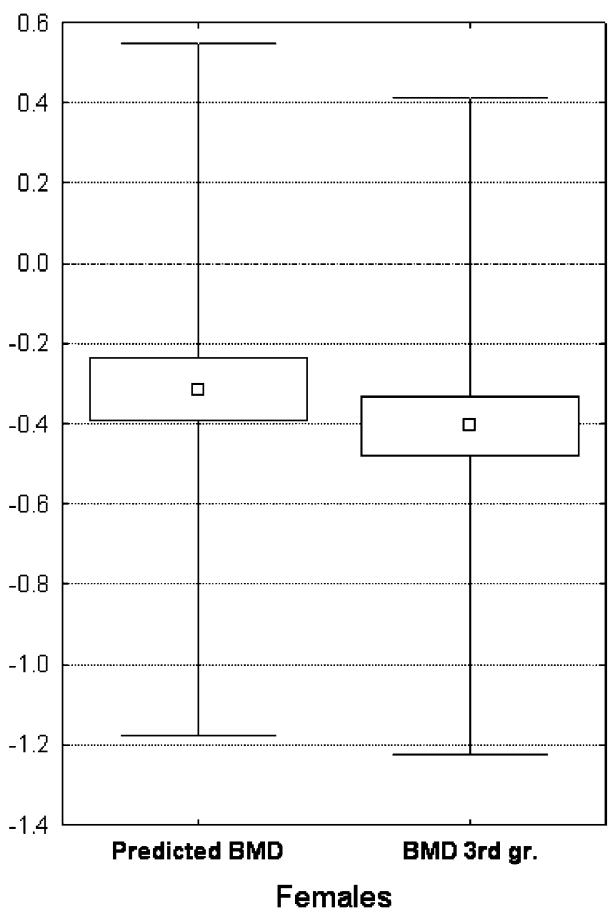


fitting curves, we found full overlapping of the data in the females' sample. However, this was not the case with males. The reason for this discrepancy is not obvious. It is possible that it rests on the accuracy of the model's prediction in two sexes. The best-fitting and most parsimonious models of age-related changes of BMD in the crosssectional sample were different between males and females. Although age explained between 48 and $58 \%$ of the BMD variation in females, it explained only $20-29 \%$ in males. Body height and weight together explained $6.5 \%$ of the residual variance for males and $4.6 \%$ for females. Other covariates showed no significant influence on age-adjusted BMD variation. The standard errors of parameter estimates in the females' sample were also generally lower, supporting our assumption that the discrepancy in the accuracy of the curve fitting may cause the difference between the expected and observed BMD in males.

In the present study, the three-interval linear mathematical function was the best-fitting model of BMD-total loss in the cross-sectional sample for females. More specifically, it showed that in females the peak bone mass is attained on the average at age 44, and then it decreases sharply with age (Fig. 3). This threshold closely matches the data obtained from cross-sectional studies by several other teams on very ethnically different populations, sampled from the various regions of Europe and Asia [14, 39, 40]. However, our present study also suggests the existence of an additional threshold (at age 60), with a much slower reduction in BMD afterwards. The slower reduction of BMD after age 60 may reflect an individual's development stage or a population tendency connected to possible selective mortality associated with lower BMD. In our sample the follow-up mortality data for an 8-year period (1994-2002) showed that the average age of death for women was 67.4 and for men 63.8. Unfortunately, insufficient mortality data did not allow us to validate the hypothesis of selective mortality associated with low BMD.

In our sample the age of peak bone mass observed in females was very similar to what was found in several other studies on various skeletal sites including the hand [14, 39, 40], but it contradicts the previous data of others [11, 4143] regarding the peak bone mass. Despite two longitudinal studies that showed that BMD in a distal site increases up to age 50 in males [27] and females [17], our results support the notion $[33,44]$ that bone loss starts during the third decade of life. This discrepancy might be explained by differences in the evaluation methods, the length of the follow-up, different sites of BMD measure, or variations in the population. It is unlikely that hand BMD in females increases until the middle of the fifth decade of age, i.e., perimenopausal age. The four-interval model for COMP in females fits well the pattern of remodeling of the bone, as expected from the abundant data from the literature [e.g.,
45, 46]. As seen in Fig. 3, there is an increase of bone density up to age 31 ; after that the COMP remains unchanged until age 48 (the mean age of menopause in the present population); then it sharply decreases until age 60 (menopausal bone loss). After age 60 the rate of bone loss slows down. Those patterns (four-interval linear model) of age-related changes of hand compacta BMD in women, as well as the pattern of age-related compacta BMD change in men (three-interval model) were almost identical to the pattern of bone mass change described by Compston [45] and by Riggs and Melton [46]. We believe that our present study provides an accurate model of age fitting, because we used a precise method of BMD evaluation and examined a sample with a wide range of ages of individuals measured. An additional strength of our study is the longest follow-up period ( 8 years). Therefore, we support the notion that bone loss begins during the third decade of life [33, 44].

At any age, the average BMD in males was higher than in females in both cross-sectional and longitudinal samples. In the longitudinal observations, during the fourth to sixth decades of life the average BMD loss was higher in males than in females (Fig. 4). During the second decade BMD loss was virtually similar in both sexes and in the seventh and eighth decades it was higher in females. We noted some discrepancies regarding the increase of BMD during the third decade of life in females, as can be seen in Fig. 3 (cross-sectional study) and virtually no average BMD change in the same age group, as shown in Fig. 4. We also noted that in both sexes the mean BMD loss predicted from the cross-sectional study was lower than observed in the longitudinal study (Fig. 5). A possible explanation is that the younger generations probably have a relatively lower peak bone mass than the older ones. Our finding of a significant difference in the mean BMD value between individuals the same age (on the day of data collection) belonging to different samples supports this assumption. Consequently, the actual rate of BMD loss, as measured in the longitudinal study, could be higher than we expected from the cross-sectional data.

This study had several limitations. First, we had only two points in time to examine the longitudinal aspect of BMD loss in the cohort. Ideally, we would have preferred several points of BMD evaluation over time to stabilize the pattern of change in BMD. Second, the sample size, of repeatedly measured individuals, was relatively small (the follow-up rate was less than $50 \%$ ). This can be explained by migration to the urban areas, probably due to significant political and socioeconomic changes in Russia between 1994 and 2002. In addition, 65 individuals from families that continued to live in the region died. The third limitation is that our longitudinal sample includes very few individuals aged over 70 and there were no individuals aged over 
77. This makes it very difficult to compare our results with other longitudinal studies, all of which were performed on an aged population. An additional limitation was that we were able to determine the peak bone mass age only roughly, with an error attributed to the maximum likelihood estimate method.

In conclusion, our study is one of the first to describe BMD change in a longitudinal, population-based study comprising both sexes from the age of 18 to 77 years. We found that digital radiographic densitometry is an easy and reliable method for assessing the mineral status at a peripheral site. It represents a low-cost and low-radiation tool for axial BMD measurements, and it is very suitable for epidemiological studies. BMD at the hands continues to fall with age in elderly women and men in this populationbased cohort. Men continue to lose BMD at all ages after the first half of the third decade, but their BMD remains higher than that of women. The most parsimonious and best-fitting piecewise linear models of age-related changes of hand BMD had a prediction value that was higher in females than in males $\left(R^{2}=0.48-0.58\right.$ vs $R^{2}=0.20-0.29$, correspondingly). The compacta BMD is more sensitive to changes in age than the total in both sexes. BMD loss in the peripheral skeleton is higher in males than in females aged 30-59, but afterwards it reverses. The highest loss in both sexes was at ages 50-59. There was good correspondence between the BMD predicted from the cross-sectional data and the BMD observed in the repeated measurement 8 years later in females, but not in males. We explained this discrepancy by better model fitting in women in comparison to men.

Acknowledgment This study was performed in partial fulfillment of the master degree requirements of G. Bigman and R. Matias. This study was supported by a grant from the Israeli National Science Foundation-"Academia", no. 1042-04 and International Osteoporosis Foundation.

\section{References}

1. Keen RW (2003) Burden of osteoporosis and fractures. Curr Osteoporos Rep 1:66-70

2. McCreadie B, Goldstein SA (2000) Biomechanics of fracture: is bone mineral density sufficient to assess risk? J Bone Miner Res 15:2305-2308

3. Miller PD (2005) Bone density and markers of bone turnover in predicting fracture risk and how changes in these measures predict fracture risk reduction. Curr Osteoporos Rep 3:103-110

4. Ammann P, Rizzoli R (2003) Bone strength and its determinants. Osteoporos Int 14(Suppl 3):S13-S18

5. Miller P, Bonnick S, Rosen C (1996) Consensus of an international panel on the clinical utility of bone mass measurements in the detection of low bone mass in the adult population. Calcif Tissue Int 58:207-214

6. Kleerekoper M, Nelson DA, Flynn MJ et al (1994) Comparison of radiographic absorptiometry with dual-energy x-ray absorptiom- etry and quantitative computed tomography in normal older black and white women. J Bone Miner Res 9:1745-1749

7. Takada M, Engelke K, Hagiwara S et al (1997) Assessment of osteoporosis: comparison of radiographic absorptiometry of the phalanges and dual X-ray absorptiometry of the radius and lumbar spine. Radiology 202:759-763

8. Bouxsein ML, Michaeli DA, Plass DB et al (1997) Precision and accuracy of computed digital absorptiometry for assessment of bone density of the hand. Osteoporos Int 7:444-449

9. Reed MR, Murray JR, Abdy SE, Francis RM, McCaskie AW (2004) The use of digital X-ray radiogrammetry and peripheral dual energy X-ray absorptiometry in patients attending fracture clinic after distal forearm fracture. Bone 34:716-719

10. Hagiwara S, Engelke K, Takada M, Yang SO, Grampp S, Dhillon MS, Genant HK (1998) Accuracy and diagnostic sensitivity of radiographic absorptiometry of the second metacarpal. Calcif Tissue Int 62:95-98

11. Baran DT (1999) Determinants of maintenance of bone mass. In: Rosen CJ, Glowacki J, Bilezikian JP (eds) The aging skeleton. Academic, San Diego, CA, pp 137-144

12. Kaji H, Kosaka R, Yamauchi M et al (2005) Effects of age, grip strength and smoking on forearm volumetric bone mineral density and bone geometry by peripheral quantitative computed tomography: comparisons between female and male. Endocr J 52:659666

13. Kalichman L, Cohen Z, Kobyliansky E et al (2002) Interrelationship between bone aging traits and basic anthropometric characteristics. Am J Hum Biol 14:380-390

14. Malkin I, Karasik D, Livshits G et al (2002) Modelling of agerelated bone loss using cross-sectional data. Ann Hum Biol 29:256-270

15. Guthrie JR, Ebeling PR, Hopper JL et al (1998) A prospective study of bone loss in menopausal Australian-born women. Osteoporos Int 8:282-290

16. Sowers M, Crutchfield M, Bandekar R et al (1998) Bone mineral density and its change in pre- and perimenopausal white women: the Michigan Bone Health Study. J Bone Miner Res 13:1134 1140

17. Chapurlat RD, Gamero P, Sornay-Rendu E et al (2000) Longitudinal study of bone loss in pre- and perimenopausal women: evidence for bone loss in perimenopausal women. Osteoporos Int $11: 493-498$

18. Bainbridge KE, Sowers MF, Crutchfield M et al (2002) Natural history of bone loss over 6 years among premenopausal and early postmenopausal women. Am J Epidemiol 156:410 417

19. Davis JW, Ross PD, Wasnich RD et al (1991) Long-term precision of bone loss rate measurements among postmenopausal women. Calcif Tissue Int 48:311-318

20. Sowers M, Clark K, Wallace R et al (1991) Prospective study of radial bone mineral density in a geographically defined population of postmenopausal Caucasian women. Calcif Tissue Int 48:232239

21. Greenspan S, Maitland LA, Myers ER et al (1994) Femoral bone loss progresses with age: a longitudinal study in women over age 65. J Bone Miner Res 9:1959-1965

22. Ensrud KE, Palermo L, Black DM et al (1995) Hip and calcaneal bone loss increase with advancing age: longitudinal results from the study of osteoporotic fractures. J Bone Miner Res 10:17781787

23. Kado DM, Browner WS, Blackwell T et al (2000) Rate of bone loss is associated with mortality in older women: a prospective study. J Bone Miner Res 15:1974-1980

24. Melton LJ 3rd, Atkinson EJ, O'Connor MK et al (2000) Determinants of bone loss from the femoral neck in women of different ages. J Bone Miner Res 15:24-31 
25. Davis JW, Ross PD, Vogel JM et al (1991) Age-related changes in bone mass among Japanese-American men. Bone Miner 15:227236

26. Vogel JM, Davis JW, Nomura A et al (1997) The effects of smoking on bone mass and the rates of bone loss among elderly Japanese-American men. J Bone Miner Res 12:1495-1501

27. Khosla S, Melton LJ 3rd, Atkinson EJ et al (2001) Relationship of serum sex steroid levels to longitudinal changes in bone density in young versus elderly men. J Clin Endocrinol Metab 86:35553561

28. Jones G, Nguyen T, Sambrook P et al (1994) Progressive loss of bone in the femoral neck in elderly people: longitudinal findings from the Dubbo osteoporosis epidemiology study. BMJ 309:691-695

29. Dennison E, Eastell R, Fall CH et al (1999) Determinants of bone loss in elderly men and women: a prospective population-based study. Osteoporos Int 10:384-391

30. Hannan MT, Felson DT, Dawson-Hughes B et al (2000) Risk factors for longitudinal bone loss in elderly men and women: the Framingham Osteoporosis Study. J Bone Miner Res 15:710-720

31. Emaus N, Berntsen GK, Joakimsen R et al (2006) Longitudinal changes in forearm bone mineral density in women and men aged 45-84 years: the Tromso Study, a population-based study. Am J Epidemiol 163:441-449

32. Henry YM, Fatayerji D, Eastell R (2004) Attainment of peak bone mass at the lumbar spine, femoral neck and radius in men and women: relative contributions of bone size and volumetric bone mineral density. Osteoporos Int 15:263-273

33. Emaus N, Berntsen GK, Joakimsen R et al (2005) Longitudinal changes in forearm bone mineral density in women and men aged 25-44 years: the Tromso study: a population-based study. Am J Epidemiol 162:633-643

34. El'chinova GI, Znichenko RA, Ginter EK (2003) Time dynamics of ethnic assortativeness in Tsivil'sk Raion of Chuvashia. Russ J Genet 39:461-462
35. Livshits G, Karasik D, Kobyliansky E (2002) Complex segregation analysis of the radiographic phalanges bone mineral density and their age-related changes. J Bone Miner Res 17:152-161

36. Emaus N, Berntsen GK, Joakimsen R et al (2005) Bone mineral density measures in longitudinal studies: the choice of phantom is crucial for quality assessment. The Tromso study, a populationbased study. Osteoporos Int 16:1597-1603

37. StatSoft, Statistica for Windows (Computer program manual) (2000) Tulsa, OK, USA

38. Malkin I, Ginsburg E (2003) Program package for Mendelian analysis of pedigree data (MAN, Version 6 technical report). Tel Aviv University, Department of Anatomy and Anthropology

39. Hyldstrup L, Nielsen SP (2001) Metacarpal index by digital X-ray radiogrammetry: normative reference values and comparison with dual X-ray absorptiometry. J Clin Densitom 4:299-306

40. Toledo VA, Jergas M (2006) Age-related changes in cortical bone mass: data from a German female cohort. Eur Radiol $16: 811-817$

41. Mazess RB (1982) On aging bone loss. Clin Orthop 165:239-252

42. Kimmel DB (1990) An evaluation of existing normal iliac trabecular bone volume (bv/tv) data. In: Takahashi HE (ed) Bone morphometry: the fifth international congress on bone morphometry. Nishimura, Japan, pp 208-215

43. Rodin A, Murby B, Smith MA et al (1990) Premenopausal bone loss in the lumbar spine and neck of femur: a study of 225 Caucasian women. Bone 11:1-5

44. Seeman E (2002) Pathogenesis of bone fragility in women and men. Lancet 359:1841-1850

45. Compston JE (1995) Review article: osteoporosis, corticosteroids and inflammatory bowel disease. Aliment Pharmacol Ther 9:237250

46. Riggs BL, Melton LJ (1992) Involutional osteoporosis. In: Evans JC, Williams TF (eds) Oxford textbook of geriatric medicine. Oxford University Press, New York 